Proceedings - 19th International Conference - IEEE/EMBS Oct. 30 - Nov. 2, 1997 Chicago, IL. USA

\title{
SEGMENTATION OF PATHOLOGY MICROSCOPIC IMAGES
}

\author{
Hui Zhu, Francis H. Y. Chan*, F. K. Lam, and K. Y. Lam \\ Department of Electrical \& Electronic Engineering, The University of Hong Kong, Hong Kong \\ Department of Pathology, The University of Hong Kong, Hong Kong \\ *E-mail: fhychan@eee.hku.hk
}

\begin{abstract}
The light microscopic analysis of the number and shape of cells in pathology is important for the diagnosis and assessment of clinical behavior of disease conditions. The fundamental step of this work is to separate the cells from the background. To segment objects from such uneven background images, a fixed threshold is not suitable. We have proposed a new adaptive thresholding method using variational theory [1]. In this paper, this method is introduced to segment pathological images under light microscope. The comparison of the segmentation results of our method and Otsu's [2] thresholding method shows the advantage of our method.
\end{abstract}

\section{INTRODUCTION}

To diagnose and assess the behavior of many diseases, microscopic image analysis is important [3]. This is a heave and complicated work for the pathologists, both time consuming and expensive. No matter what stereology methods are used to analyze these images [4,5], a basic image segmentation step must be adopted first to separate the cells from their background. Thresholding methods [6] are often used to deal with this problem. In such microscopic images, the backgrounds are usually uneven, and adaptive (or local) threshold should be used instead of global threshold to give better result.

In this paper, we will first introduce an adaptive thresholding method we recently developed [1]. Then the method is used to segment an image from an immunohistochemical stained section. The result is compared with the result from Otsu's global thresholding method [2].

\section{THE ADAPTIVE THRESHOLDING METHOD}

We can view the gray level image as a 2-D surface. In order to construct an adaptive threshold surface, we aim to find a smooth surface which meets the image surface at the points where the gradients of the image are local highest [1]. These points are the boundaries of the objects. Then the objects can be segmented out adaptively from the background.
Let $I(x, y)$ be an image which is to be segmented by thresholding, and $G(x, y)$ is the normalized gradient magnitude of $I(x, y)$.

$$
G(x, y)=\frac{|\nabla I(x, y)|}{\max _{x, y}(|\nabla I(x, y)|)}
$$

$T(x, y)$ is the adaptive threshold to be found by which the image is segmented. $I(x, y)$ and $T(x, y)$ are two 2-D surfaces which intersect at positions $c=\{(x, y) \mid I(x, y)=T(x, y)\} . c$ should be where the object boundaries are. That means if $T(x, y)$ is the right threshold surface, $\int G(x, y) d H^{1}(x, y) / C$ ( $H^{1}$ is the Hausdorff measure supported by $c$, and $C$ is the number of object boundary points) should be a maximum. This necessitates in finding $T$ in a function space $\Omega$ to

$$
\min _{T \in \Omega} \int_{t} F(T) d H^{1}(x, y) / C
$$

in which, $F(T)$ is a functional of $T$ and $F(T)=-G(x, y),(x, y) \in c$. This is an ill-posed problem [7].

To solve the problem, a penalty term is introduced into the object function for regularization. Then the object function to be minimized is

$$
\frac{1}{C} \int F(T) d H^{1}(x, y)+\lambda \int\left(\left|\frac{\partial T}{\partial x}\right|^{2}+\left|\frac{\partial T}{\partial y}\right|^{2}\right) d x d y
$$

where $\lambda$ is the regularization parameter. Minimizing (2) is equal to solving the following Poisson equation:

$$
\begin{cases}\nabla^{2} T(x, y)=\alpha \frac{\delta F(T)}{\delta T}, & \text { if }(x, y) \in c \\ \nabla^{2} T(x, y)=0, & \text { else }\end{cases}
$$

Here, $\alpha=1 / 2 \lambda$. Because $F(T)$ relates to $c, F(T)$ can be written as $F(x(T), y(T))$. By the chain rule,

$$
\frac{\delta F}{\delta T}=F_{x} \cdot \frac{\partial x}{\delta T}+F_{y} \cdot \frac{\partial y}{\delta T}=F_{x} / \frac{\delta T}{d x}+F_{y} / \frac{\delta T}{d y}
$$

Note that here $T$ is a function, $\delta T$ is the variation of $T$.

Now we have modeled the adaptive thresholding surface as the solution of a Poisson equation (4). In practical application, only numerical solution can be obtained for the implementation of this method. Successive over-relaxation (SOR) method [8] is used here to solve this equation. The detail implementation algorithm can be found in [1]. 
Proceedings - 19th International Conference - IEEE/EMBS Oct. 30 - Nov. 2, 1997 Chicago, IL. USA

\section{RESULTS AND DISCUSSIONS}

An image from immunohistochemical stained (MIB-1) esophageal carcinoma taken by a CCD camera is shown in Fig.1. It can be seen that the background gray level is uneven. The ideal segmentation result to such images is that all the cells are segmented and isolated. Fig.2 is the segmentation result of our adaptive thresholding method to Fig.1. Fig. 3 is the result by Otsu's method to Fig. 1 . We can see that, in Fig.3, while the separated cells in region A are connected, those in regions $\mathrm{B}$ and $\mathrm{C}$ are missing or incomplete. Raising the threshold, more cells in regions $\mathrm{B}$ and $\mathrm{C}$ can be seen but more cells will connected to a block in A. On the contrary, if we lower the threshold, the cells in region $\mathrm{A}$ can be isolated, but more cells will missing in other parts of the image. This means there is no optimal global threshold to segment the image satisfactory. Our method is tolerant to such a situation. Because the threshold is not a constant, but a surface adaptively changing with the background, different threshold is set at different places according to their local image information. So the result is much more robust and consistent.

We have pointed out that this adaptive thresholding method is particularly suitable for images containing many objects [1]. This is a typical situation encountered in light microscopic images in pathology.

\section{REFERENCES}

[1] F.H.Y.Chan, F.K.Lam, \& Hui Zhu, "Adaptive thresholding by variational method," to appear in IEEE Trans. Image Processing.

[2] N.Otsu, "A threshold selection method from gray-level histogram," IEEE Trans. Syst., Man, Cybern., vol.8, pp.62-66, 1979.

[3] K.Y.Lam, S.Y.K.Law, M.K.P.So, M.Fok, L.T.Ma, \& J.Wong, "Prognostic implication of proliferative markers MIB-1 and $\mathrm{PCl} 0$ in esophageal squamous cell carcinoma", Cancer, vol.77, pp7-13, 1996.

[4] H.J.G.Gundersen, T.F.Bendtsen, L.Korbo, N.Marcussen, A.Moller, K.Nielsen, J.R.Nyengaard, B.Pakkenberg, F.B.Sorensen, A.Vesterby \& M.J.West, "Some new, simple and efficient stereological methods and their use in pathological research and diagnosis," APMIS, vol.96, pp.379-394, 1988.

[5] H.J.G.Gundersen, P.Bagger, T.F.Bendtsen, S.M.Evans, L.Korbo, N.Marcussen, A.Moller, K.Nielsen, J.R.Nyengaard, B.Pakkenberg, F.B.Sorensen, A.Vesterby \& M.J.West, "The new stereological tools: disector, fractionator, nucleartor and point sampled intersepts and their use in pathological research and diagnosis," APMIS, vol.96, pp.857-881, 1988.
[6] P.K.Sahoo, S.Soltani, A.K.C.Wong \& Y.C.Chen, "A survey of thresholding techniques," Comput. Vision, Graph., Image Processing, vol.41, pp.233-260, 1988.

[7] A.N.Tikhonov \& V.Y.Arsenin, Solutions of ill-posed problems, New York: Winston, 1977.

[8] W.H. Press, S.A. Teıkolsky, W.T .Vetterling \& B.P. Flannery, Numerical Recipes in C: The Art of Scientific Computing, $2^{\text {nd }}$ ed., Cambridge University Press, 1992.

A

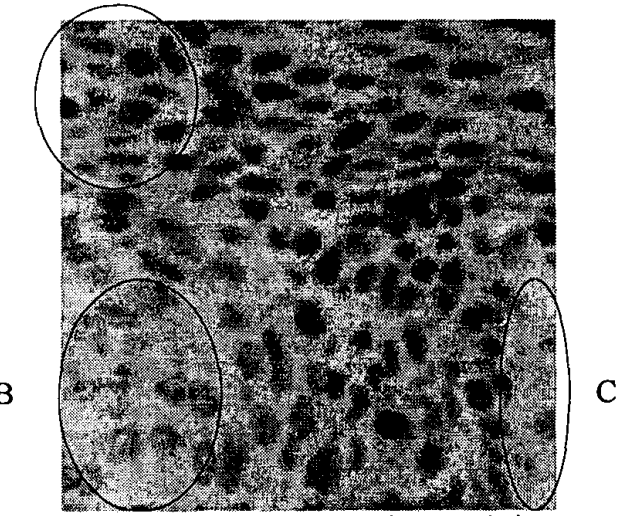

Fig.1 An esophageal cancer microscopic image

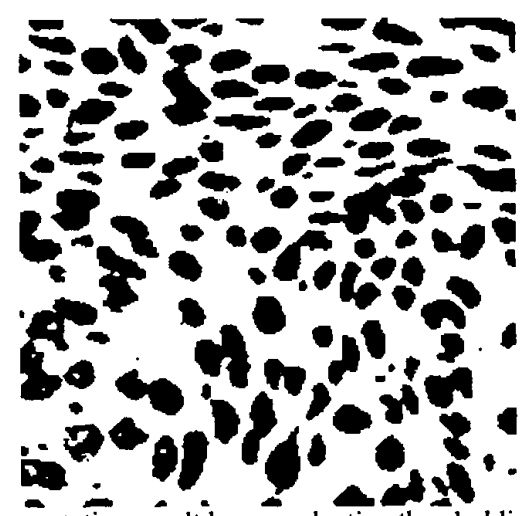

Fig.2 Segmentation result by our adaptive thresholding method

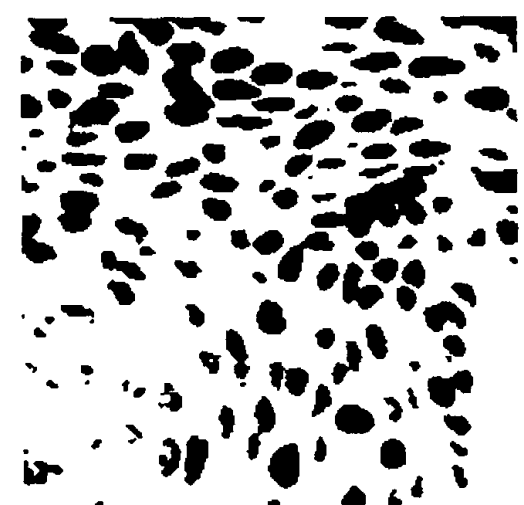

Fig.3 Segmentation result by Otsu's method 